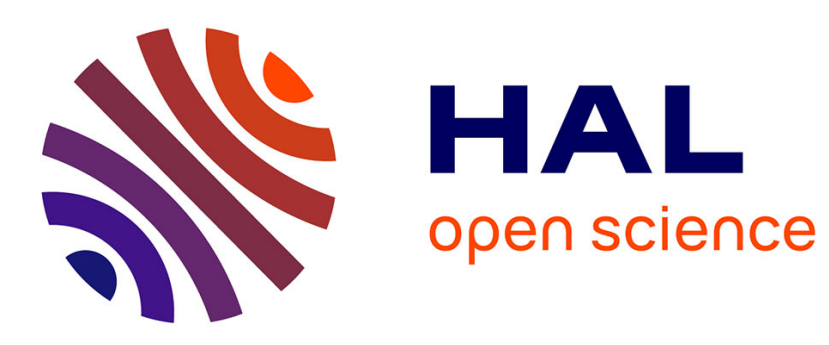

\title{
Driving Species Competition in a Light-limited Chemostat
}

\author{
Francis Mairet, Rafael Munoz Tamayo, Olivier Bernard
}

\section{To cite this version:}

Francis Mairet, Rafael Munoz Tamayo, Olivier Bernard. Driving Species Competition in a Lightlimited Chemostat. 9th IFAC Symposium on Nonlinear Control Systems (NOLCOS), Sep 2013, Toulouse, France. hal-00852258

\section{HAL Id: hal-00852258 \\ https://hal.inria.fr/hal-00852258}

Submitted on 20 Aug 2013

HAL is a multi-disciplinary open access archive for the deposit and dissemination of scientific research documents, whether they are published or not. The documents may come from teaching and research institutions in France or abroad, or from public or private research centers.
L'archive ouverte pluridisciplinaire HAL, est destinée au dépôt et à la diffusion de documents scientifiques de niveau recherche, publiés ou non, émanant des établissements d'enseignement et de recherche français ou étrangers, des laboratoires publics ou privés. 


\title{
Driving Species Competition in a Light-limited Chemostat *
}

\author{
Francis Mairet* Rafael Muñoz-Tamayo* Olivier Bernard*,** \\ * BIOCORE-INRIA, BP93, 06902 Sophia-Antipolis Cedex, France \\ (e-mail: \{francis.mairet, rafael.munoz_tamayo, \\ olivier.bernard\}@inria.fr) \\ ** LOV-UPMC-CNRS, UMR 7093, Station Zoologique, B.P. 2806234, \\ Villefranche-sur-mer, France
}

\begin{abstract}
In this paper, we tackle the problem of microalgae selection in a continuous photobioreactor where microalgae growth is limited by light. We propose a closed-loop control for selecting, for a given range of light intensity, the strain with the maximum growth rate from the microalgae population. In particular, we are interested in strains with high growth rate for high light intensity, i.e.strains with high resistance to photoinhibition. Firstly, we recall the framework of the light-limited chemostat. Then, we propose a nonlinear adaptive control which regulates the light intensity at the bottom of the photobioreactor in monoculture. This light is of particular interest as it defines the winner of the competition in a multispecies culture operated in open-loop mode. Finally, we show that the proposed controller allows the selection of a strain of interest in the case of a culture with $n$ species.
\end{abstract}

Keywords: Nonlinear adaptive control, Photobioreactor, Light competition, Microalgae, Species selection.

\section{INTRODUCTION}

Microalgae culture has emerged as a promising system for the production of high value compounds in the industry of food, cosmetics and energy (Spolaore et al., 2006; Chisti, 2007; Wijffels and Barbosa, 2010). Despite its high potential, there is still a long way before reaching economic and environmental viability for large scale production.

In this context, the selection of species of interest is a way to increase productivity, similarly to what have been done in agriculture. This poses an interesting challenge: in a culture consisting of different species, how to select the species that better perform with respect to a desired property?

Here, our objective is to propose principles in order to select microalgae of interest in light-limited chemostat cultures. Note that this selection process can be done at two scales:

- Choosing the best species from a pool of species. Actually, microalgae presents a huge diversity which has not been exploited.

- For one species, choosing the best strain. Here, the idea is to exploit intra-species diversity in order to select a particular trait.

In the following, we will use abusively the terms strain and species selection to refer to both cases.

More precisely, we want to select, from the microalgae population, the species with the maximum growth rate

\footnotetext{
* This work benefited from the support of the Facteur 4 research project founded by the French National Research Agency (ANR).
}

for a given range of light intensity. For example, we are interested in species with high growth rate for high light intensity, i.e., with high resistance to photoinhibition. When photoinhibition occurs, the photosynthetic rate decreases as result of light-induced damage to photosystems. Therefore, this phenomenon can severely reduce biomass production. The final objective will be to use the different species that we have selected in order to optimize microalgae production.

In open-loop culture, selection of species of interest is delicate. Actually, such process will select a species according to a criteria inherent to the system, which is not necessarily of interest. Closed loop controls have been proposed to drive microorganisms competition in substratelimited chemostat (e.g. Masci et al. (2009); Sbarciog and Vande Wouwer (2012) for anaerobic digestion). In Masci et al. (2008), a periodic substrate stress is imposed to a chemostat in order to select species with respect to a new criterion that involves the capacity of the microorganisms to increase its internal substrate storage.

Here, we design an adaptive controller that can be exploited to drive microalgae selection. The controller developed is based on the work of Mailleret et al. (2004).

The paper is organized as follows. We present firstly a brief state of the art of the theory of ecological competition, in particular for light limited culture (Huisman and Weissing, 1994). This framework will be the basis of our development. In Section 3, we propose an adaptive controller which regulates the light at the bottom of the water column in monoculture. Then, we show that the proposed controller allows the selection of a strain of interest in the 
case of a culture with $n$ species. Finally, we illustrate our approach with numerical simulations.

\section{THEORETICAL ASPECTS OF COMPETITION}

\subsection{Competition by substrate limitation}

The competition of microorganisms limited by a substrate has been widely studied both experimentally and theoretically (Smith and Waltman, 1995). The Competitive Exclusion Principle states that if $n$ competing species are introduced in a chemostat and compete for one single substrate, only one species will stay in the chemostat, while the others $n-1$ species will disappear. The study showed that the winner of the the competition could be predicted; it is the species that can grow at a rate equal to the dilution rate with the smallest amount of limiting nutrient $s_{i}$. This principle was validated experimentally (Hansen and Hubbell, 1980).

\subsection{Competition for light}

Competition for light occurs for microalgae through the light gradient created by biomass shading. Thus, the theory developed for substrate does not hold in this case. This section illustrates the principles of competition for light, based on the research work developed by Jef Huisman and co-workers (Huisman and Weissing, 1994; Weissing and Huisman, 1994; Stomp et al., 2004; Gerla et al., 2011). Originally developed for natural systems (lake, ocean...), this theoretical approach can be adapted and exploited in the framework of microalgae culture systems (photobioreactor or raceways). ${ }^{1}$

Modeling monoculture growth Let us consider a mixed water column of length L devoted to the continuous culture of microalgae $x$. We assume that the system is completely homogeneous, i.e., the concentration of microalgae is the same in all the points of the reactor. Due to light absorption and diffusion by the algae, a spatial gradient of light occurs: the light intensity in the reactor decreases along the depth.

Let us assume that the absorption of light in the water column follows the Lambert-Beer law. Thus, for a given depth $z$, the corresponding light intensity $I(x, z)$ satisfies

$$
I(x, z)=I_{\text {in }} \exp (-a x z),
$$

where $I_{i n}$ is the incident light, and $a$ is the coefficient of attenuation due to microalgae. The light at the bottom of the water column is called $I_{\text {out }}(x)=I(x, L)$.

We consider here that the growth of microalgae is only limited by light, and it is defined by the specific growth rate $\mu(I)>0, \forall I>0$. The total growth $G$ of microalge is obtained by integrating the local growth over depth:

$$
G(x)=\frac{x}{L} \int_{0}^{L} \mu(I(x, z)) d z
$$

\footnotetext{
1 In this subsection, results are given without proof. For more details, readers should refer to the original works.
}

Given Equation (1), we obtain

$$
G(x)=\frac{1}{a L} \int_{I_{\text {out }}(x)}^{I_{\text {in }}} f(I) d I
$$

with $f(I)=\mu(I) / I$.

Given a constant dilution rate $u^{2}$, the dynamic evolution of the microalgae concentration $x$ is therefore given by

$$
\dot{x}=G(x)-u x \text {. }
$$

Model analysis In the following, we consider a constant light supply $I_{i n}$. The asymptotic behavior of the model depends on the growth rate:

- Increasing growth rate (Huisman and Weissing, 1994; Weissing and Huisman, 1994)

If $u<\mu\left(I_{i n}\right)$, Equation (4) has one non-trivial equilibrium, which is globally stable.

For example, assuming a kinetics of Michaelis-Menten type, the growth rate writes:

$$
\mu(I)=\bar{\mu} \frac{I}{I+K_{s}} .
$$

The total growth $G$ is obtained by integration along the depth:

$$
G(x)=\frac{\bar{\mu}}{a L} \ln \left(\frac{I_{\text {in }}+K_{s}}{I_{\text {out }}(x)+K_{s}}\right) .
$$

The nontrivial equilibrium of (4) is obtained by solving

$$
\frac{\bar{\mu}}{a L} \ln \left(\frac{I_{i n}+K_{s}}{I_{i n} \exp \left(-a x^{\star} L\right)+K_{s}}\right)=u x^{\star},
$$

where $x^{\star}$ is the biomass concentration at steady state. This allows to define the light at the bottom of the water column at equilibrium $I_{\text {out }}^{\star}=I_{\text {in }} \exp \left(-a x^{\star} L\right)$.

\section{- Nonmonotone growth rate (photoinhibition)}

We now consider the case where the specific growth rate has one maximum (e.g. a Haldane function). It follows that Equation (4) can have one (globally stable) or two nontrivial equilibria. In this last case, one is locally stable and the other unstable, the trivial equilibrium is also locally stable. This corresponds to a strong Allee effect: at low concentration, the specific growth rate increases with biomass concentration (self-shading reduces the negative impact of photoinhibition). Nevertheless, below a threshold biomass concentration (corresponding to the unstable equilibrium), the biomass goes extinct (Gerla et al., 2011).

If a Haldane function is used to represent the growth rate:

$$
\mu(I)=\bar{\mu} \frac{I}{K_{s I}+I+I^{2} / K_{i I}},
$$

the total growth $G$ obtained by integration over depth is written, considering that $K_{i I}<4 K_{s I}$ (Bernard et al., 2009; Bernard, 2011):

\footnotetext{
2 Initially, Huisman and co-workers used a loss rate which gathers dilution, mortality, predation... In a chemostat, we assume that the loss is mainly due to dilution.
} 


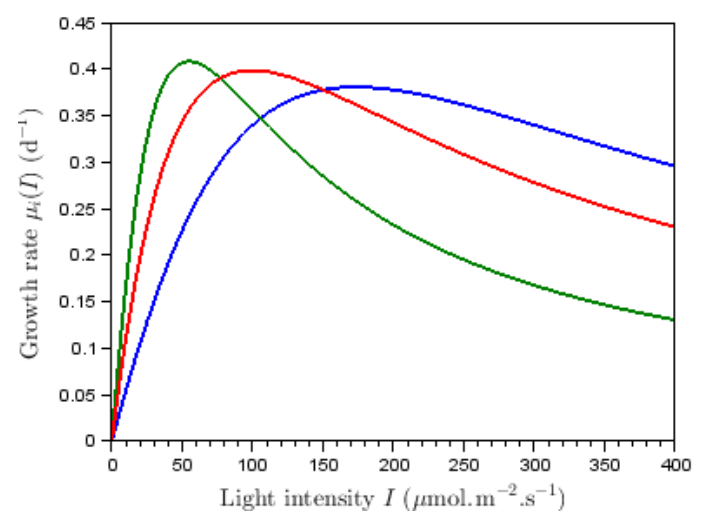

Fig. 1. Specific growth rate $\mu_{i}(I)$ as a function of light intensity $I$ for three different microalgal species.

$$
\begin{gathered}
G(x)=\bar{\mu} \frac{2 K_{i I}}{a L \sqrt{\Delta}}\left[\arctan \left(\frac{2 I_{\text {out }}(x)+K_{i I}}{\sqrt{\Delta}}\right)\right. \\
\left.-\arctan \left(\frac{2 I_{\text {in }}+K_{i I}}{\sqrt{\Delta}}\right)\right]
\end{gathered}
$$

where $\Delta=K_{i I}\left(4 K_{s I}-K_{i I}\right)$.

Competition Let us consider now the case of a culture under constant illumination with $n$ microalgal populations (denoted $\left.x_{i}\right)$ competing for light. Denote $X=\left(x_{1}, \ldots, x_{n}\right)$.

The light at depth $z$ is now

$$
I(X, z)=I_{\text {in }} \exp \left(-\left(\sum_{i=1}^{n} a_{i} x_{i}\right) z\right) .
$$

Given this light gradient, the following dynamical system represents the competition of the $n$ microalgal species:

$$
\dot{x}_{i}=G_{i}(X)-u x_{i}, i=1, \ldots, n .
$$

with

$$
\begin{aligned}
G_{i}(X) & =\frac{x_{i}}{L} \int_{0}^{L} \mu_{i}(I(X, z)) d z \\
& =\frac{x_{i}}{\left(\sum_{j=1}^{n} a_{j} x_{j}\right) L_{I_{\text {out }}(X)}} \int_{i \text { in }}^{I_{\text {in }}} f_{i}(I) d I
\end{aligned}
$$

For monotone growth rates (e.g. Monod kinetics), the dynamics of the microalgal species are interestingly related to their value $I_{o u t, i}^{*}$ in monoculture. The concentration of species $i$ will increase if the light at the bottom of the water column $I_{\text {out }}$ is such that $I_{\text {out }}>I_{\text {out }, i}^{*}$, it will decrease if $I_{\text {out }}<I_{\text {out }, i}^{*}$ and it will remain stable if $I_{\text {out }}=I_{\text {out }, i}^{*}$ (Huisman and Weissing, 1994). This property establishes the condition of exclusion between $n$ species: Under constant light, the species with the lowest $I_{\text {out }, i}^{*}$ will exclude the other species. This competitive exclusion principle for light-limited culture has been validated with chemostat experiments (Huisman et al., 1999).

For nonmonotone growth rates, this principle does not hold anymore: the issue of the competition can depend on the initial conditions if at least one of the species has two nontrivial equilibria.
Table 1. Parameter values used for simulation

\begin{tabular}{lccc} 
Species & 1 (blue) & 2 (green) & 3 (red) \\
\hline $\bar{\mu}\left(d^{-1}\right)$ & 1.7 & 1.9 & 1.12 \\
$K_{s I}\left(\mu \mathrm{mol} \cdot \mathrm{m}^{-2} \cdot s^{-1}\right)$ & 300 & 100 & 90 \\
$K_{i I}\left(\mu \mathrm{mol} \cdot \mathrm{m}^{-2} \cdot s^{-1}\right)$ & 100 & 30 & 110 \\
$a\left(\mathrm{~m}^{2} \cdot g^{-1}\right)$ & 0.3 & 0.3 & 0.3 \\
\hline
\end{tabular}

Illustration For illustration purpose, simulations of a culture with three microalgal species were performed. The specific growth rates of the three species $\left(x_{1}, x_{2}, x_{3}\right)$ are represented by Haldane functions with different parameter values (see Figure 1 and Table 1). Our goal will be to propose a procedure to separate one particulate species, in order to have strains adapted to low, mid and high light intensity. In Figure 2, a bifurcation diagram is presented. It shows the dependency of the dilution rate on the equilibria in monoculture for each species.

For a given dilution rate, if the species with the lowest $I_{\text {out }, i}^{*}$ has only one nontrivial equilibrium, this species will win the competition. Otherwise, the issue of the competition can depend on the initial conditions. For this incident light, it is observed that dilution rates below $0.34 \mathrm{~d}^{-1}$ favor the survival of the green species $x_{2}$. The blue species $\left(x_{1}\right)$ can win the competition only for dilution rates between 0.369 and $0.377 \mathrm{~d}^{-1}$. Nevertheless, in this case, the issue of the competition depends on the initial conditions. This is illustrated on Figure 3. Simulations were performed with the same dilution rate and incident light considering different sets of initial conditions. Three cases are possible: washout, selection of species 1 (blue) and selection of species 3 (red).

It is worth noting that it is difficult to select strains of interest in open-loop control. In particular, in this example, it seems very delicate to select the blue strain, even with a perfect knowledge of species properties (which is totally unrealistic): it requires a good choice of dilution rate (between $0.369 \mathrm{~d}^{-1}$ and $0.377 \mathrm{~d}^{-1}$ ) and also initial conditions. This has led us to develop a controller in order to favor the microalgal strain of interest.

\section{DRIVING COMPETITION}

Now, we propose to use the dilution rate $u$ as a control in order to regulate $I_{\text {out }}$ to a desired set-point $I_{\text {out }}^{*}$ in closed loop. In the following, we consider an undemanding assumption on the growth rate, and the availability of two measurements:

Hypothesis 1. The specific growth rate $\mu(I)$ is continuous and such that $\mu(I)>0, \forall I>0$.

Hypothesis 2. We consider that the following measurements are available:

- the light attenuation factor:

$$
y=\sum_{i=1}^{n} a_{i} x_{i}=\frac{1}{L} \ln \left(\frac{I_{\text {in }}}{I_{\text {out }}(X)}\right)
$$

- the total growth $G$

The light attenuation factor $y$ can be easily computed from light measurements at two places in the reactor. The total growth $G$ can be estimated using observer-based estimator (Bastin and Dochain, 1990; Perrier et al., 2000; Mairet 


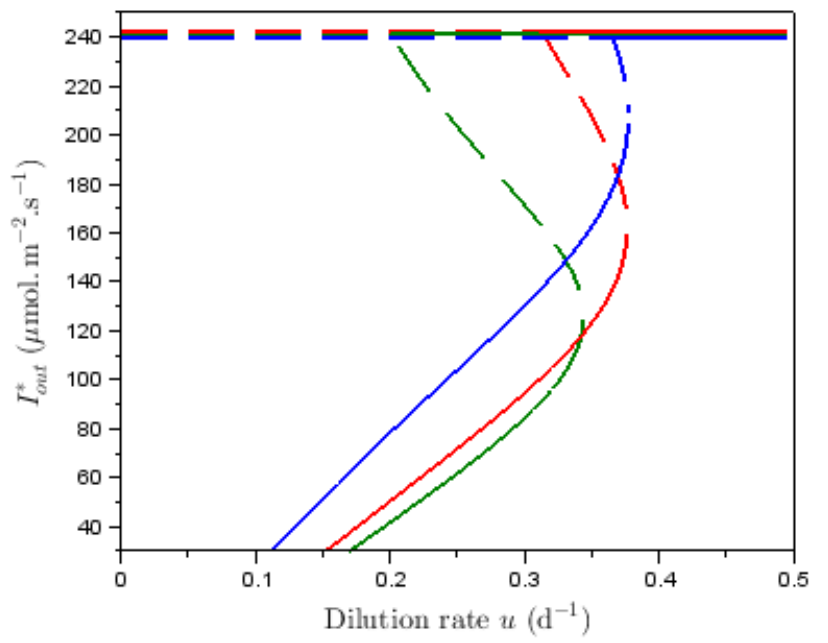

Fig. 2. Bifurcation diagram with respect to dilution rate for the three species in monoculture (with $I_{i n}=$ $\left.240 \mu \mathrm{mol} . \mathrm{m}^{-2} \cdot \mathrm{s}^{-1}\right)$. Equilibrium $x_{i}^{\star}$ is represented by its corresponding light intensity at the bottom of the water column $I_{\text {out }, i}^{\star}=I_{\text {in }} \exp \left(-a_{i} x_{i}^{\star} L\right)$ given that it is the factor that will define the issue of the competition. Solid lines: stable equilibrium, dashed lines: unstable equilibrium.
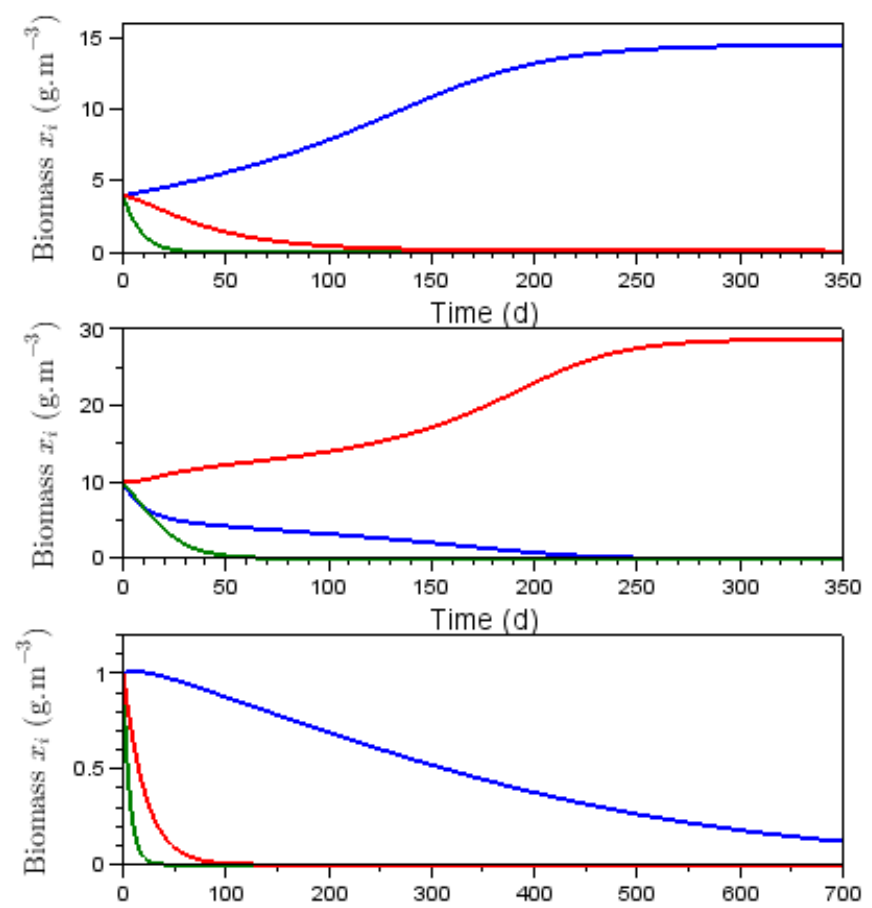

Fig. 3. Simulations of competition in open-loop for different sets of initial conditions. Operating conditions: $I_{\text {in }}=240 \mu \mathrm{mol} . \mathrm{m}^{-2} \cdot \mathrm{s}^{-1}, u=0.37 d^{-1}$. The issue of competition depends on the initial conditions. et al., 2010) with oxygen measurement for example. Note that $G=\sum_{i=1}^{n} G_{i}$ in culture with $n$ species.

\subsection{Controller design in monoculture}

We propose a feedback law (based on the work of Mailleret et al. (2004)) which involves an adaptive gain $\gamma(t)$. From $I_{\text {out }}^{*}$, one can define the corresponding set-points:

$$
y^{*}=\frac{1}{L} \ln \left(\frac{I_{\text {in }}}{I_{\text {out }}^{*}}\right) \text { and } \gamma^{*}=\frac{a}{y^{*}}
$$

Theorem 3. Under Hypotheses 1 and 2, the adaptive feedback control law

$$
\left\{\begin{array}{l}
u(t)=\gamma(t) G(x) \\
\dot{\gamma}=K G(x)\left(y-y^{*}\right)\left(\gamma-\gamma_{m}\right)\left(\gamma_{M}-\gamma\right)
\end{array}\right.
$$

with $0<\gamma_{m}<\gamma^{*}<\gamma_{M}$ and $K>0$ globally stabilizes System 4 towards the positive set point $x^{*}=y^{*} / a$.

Proof. Given that $y=a x$, System (4) under control law (13) becomes:

$$
\left\{\begin{array}{l}
\dot{y}=G(x)(a-\gamma y) \\
\dot{\gamma}=K G(x)\left(y-y^{*}\right)\left(\gamma-\gamma_{m}\right)\left(\gamma_{M}-\gamma\right)
\end{array}\right.
$$

Since $G(x) \geq 0$, the set $\mathcal{R}^{+*} \times\left[\gamma_{m}, \gamma_{M}\right]$ is positively invariant (in the following, we assume initial conditions belonging to this set). Given its dynamics, one can show that $y(t) \geq y, \forall t \geq 0$, with:

$$
\underline{y}=\min \left(y(0), \frac{a}{\gamma_{M}}\right) \text {. }
$$

So we have $I_{\text {out }} \leq I_{\text {in }} \exp (-y L), \forall t \geq 0$. Using Equation (3), we deduce that $G(x)$ is lower bounded (since $f(I)>$ $0, \forall I>0)$. Thus, we can introduce the time change $t^{\prime}=\int_{0}^{t} G(\tau) d \tau$. Denoting with a prime the derivatives with respect to $t^{\prime}$, System (14) rewrites:

$$
\left\{\begin{array}{l}
y^{\prime}=a-\gamma y \\
\gamma^{\prime}=K\left(y-y^{*}\right)\left(\gamma-\gamma_{m}\right)\left(\gamma_{M}-\gamma\right)
\end{array}\right.
$$

Now consider the following Lyapunov candidate function V (Mailleret et al. (2004)):

$$
V=\int_{y^{*}}^{y} \frac{w-y^{*}}{w} d w+\int_{\gamma^{*}}^{\gamma} \frac{w-\gamma^{*}}{K\left(w-\gamma_{m}\right)\left(\gamma_{M}-w\right)} d w .
$$

The derivative of $V$ along the trajectories of System (15) is given by

$$
V^{\prime}=-a \frac{\left(y-y^{*}\right)^{2}}{y y^{*}}
$$

$V$ is a continuously differentiable, radially unbounded, positive definite function with $V^{\prime}(y, \gamma) \leq 0, \forall(y, \gamma) \in$ $\mathcal{R}^{+} \times\left[\gamma_{m}, \gamma_{M}\right]$. Moreover, one can easily check that the largest invariant set defined by $V^{\prime}=0$ is actually the set-point $\left(y^{*}, \gamma^{*}\right)$. Therefore, using Krasovskii theorem (Khalil, 2002), the set-point $\left(y^{*}, \gamma^{*}\right)$ is globally asymptotically stable.

Note that this control scheme does not require any knowledge of the growth rate or the attenuation coefficient. For the tuning of the control law, $\gamma_{M}$ can be determined according to the maximum dilution rate (allowed by technical constraints), $\gamma_{m}$ can take any value sufficiently small, 

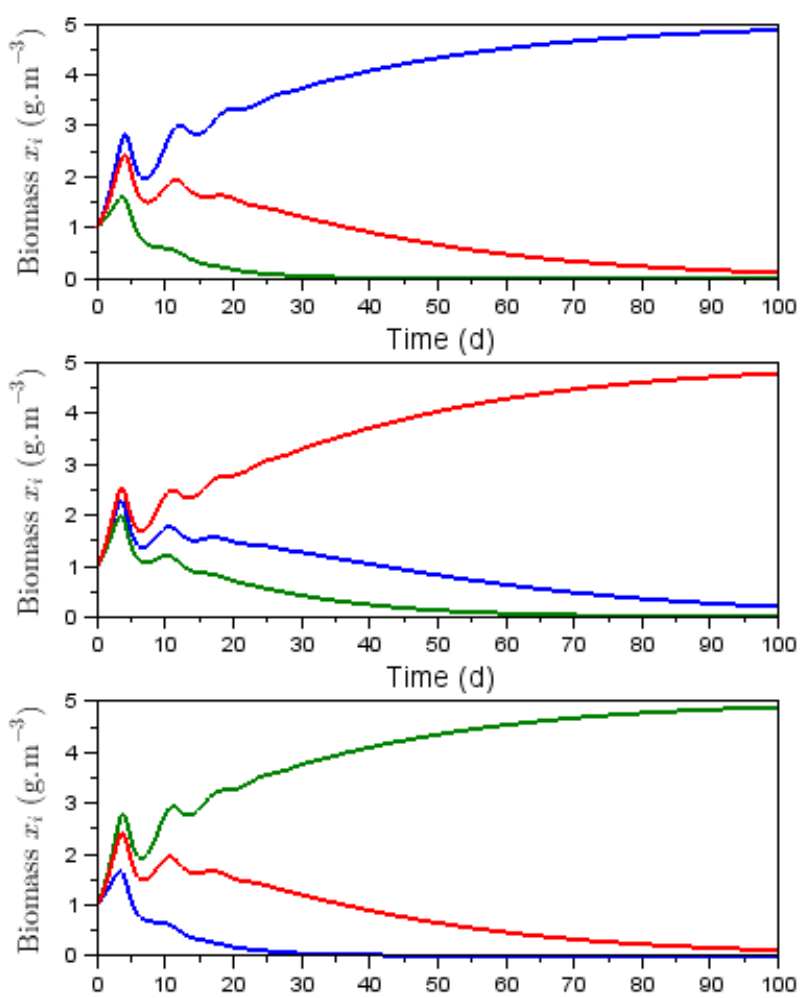

Fig. 4. Simulations of competition in closed-loop with 3 species (see Theorem 5). Operating conditions: top: $I_{\text {in }}=240 \mu \mathrm{mol} \cdot \mathrm{m}^{-2} \cdot \mathrm{s}^{-1}$, middle: $I_{\text {in }}=$ $140 \mu \mathrm{mol} \cdot \mathrm{m}^{-2} \cdot \mathrm{s}^{-1}$, bottom: $I_{i n}=70 \mu \mathrm{mol} \cdot \mathrm{m}^{-2} \cdot \mathrm{s}^{-1}$. Set-point: $x_{i}^{*}=5 \mathrm{~g} \cdot \mathrm{m}^{-3}$. Each species can be selected choosing accordingly the incident light and the setpoint $x_{i}^{*}$ (or equivalently the light at the bottom of the water column $I_{\text {out }}^{*}$ ).

and $K$ should result form a trade-off between rapidity and smoothness (a too high value can cause oscillations).

\subsection{Selection of species}

We now consider a culture with $n$ species under constant illumination, and we will show that the adaptive control law (13) can be applied in order to select the best species for a given range of light intensity, that is the strain with the maximum growth rate in this range.

Hypothesis 4. We assume:

(i) $a_{i}=a, \forall i=1, \ldots, n$

(ii) Given $I_{\text {in }}$ and $I_{\text {out }}^{*}$, there exists $i \in\{1, \ldots, n\}$ such that:

$$
\int_{I_{\text {out }}^{*}}^{I_{\text {in }}} f_{i}(I) d I>\int_{I_{\text {out }}^{*}}^{I_{\text {in }}} f_{j}(I) d I, \forall j \neq i
$$

Note that Hypothesis 4(ii) is not at all restrictive, as it only expresses that there exists one species $i$ which as a maximal integral term with respect to all the other species (in practice, it is almost impossible that two species have the same (maximal) integral term). Hypothesis 4(i) is more restrictive: the pigment content and composition can differ between species, which affects the attenuation coefficients $a_{i}$. This assumption will be discuss later.
Theorem 5. Under Hypotheses 1, 2, and 4, the adaptive control law (13) stabilizes System (11) towards the positive set point $X^{*}=\left(0, \ldots, 0, x_{i}^{*}, 0, \ldots, 0\right)$ where $x_{i}^{*}=y^{*} / a_{i}$.

\section{Proof.}

Applying control law (13) to System (11) yields to

$$
\left\{\begin{array}{l}
\dot{x}_{i}=G_{i}(X)-\gamma G(X) x_{i}, i=1, \ldots, n \\
\dot{\gamma}=K G(X)\left(y-y^{*}\right)\left(\gamma-\gamma_{m}\right)\left(\gamma_{M}-\gamma\right)
\end{array}\right.
$$

First, we prove that the control law stabilizes subsystem $(y, \gamma)$. Actually, given Hypothesis 4(i), we have $y=$ $a \sum_{j=1}^{n} x_{j}$, so we obtain the following subsystem:

$$
\left\{\begin{array}{l}
\dot{y}=G(X)(a-\gamma y) \\
\dot{\gamma}=K G(X)\left(y-y^{*}\right)\left(\gamma-\gamma_{m}\right)\left(\gamma_{M}-\gamma\right)
\end{array}\right.
$$

Using the same scheme as for the proof of Theorem 3, we can show that $y$ converges asymptotically towards $y^{*}$.

Now let us define $d_{j}=\ln \left(\frac{x_{i}}{x_{j}}\right), \forall j \neq i$, whose dynamics is written:

$$
\dot{d}_{j}=\frac{\int_{I_{\text {out }}(t)}^{I_{\text {in }}} f_{i}(I) d I-\int_{I_{\text {out }}(t)}^{I_{\text {in }}} f_{j}(I) d I}{y L}
$$

Given Hypothesis 4(ii), we have $\forall j \neq i$ :

$$
\Delta_{j}:=\int_{I_{\text {out }}^{*}}^{I_{\text {in }}} f_{i}(I)-f_{j}(I) d I>0
$$

so Equation (18) rewrites:

$$
\dot{d}_{j}=\frac{\int_{I_{\text {out }}(t)}^{I_{\text {out }}^{*}} f_{i}(I)-f_{j}(I) d I+\Delta_{j}}{y L}
$$

Now given that $y$ and $I_{\text {out }}$ converge respectively towards $y^{*}$ and $I_{\text {out }}^{*}$, and that $f_{i}(I)-f_{j}(I)$ is bounded in the neighborhood of $I_{\text {out }}^{*}$ (by Hypothesis 1 ), we have:

$$
\lim _{t \rightarrow+\infty} \dot{d}_{j}=\frac{\Delta_{j}}{y^{*} L}>0
$$

So we can finally conclude that $\lim _{t \rightarrow+\infty} d_{j}=+\infty$ and System (16) converges towards the set-point $X^{*}=$ $\left(0, \ldots, 0, x_{i}^{*}, 0, \ldots, 0\right)$.

The selection process presented in Theorem 5 is illustrated with numerical simulations (Figure 4). We use the following set of parameters for the control law: $K=1$, $\gamma_{m}=0.001 \mathrm{~m}^{3} \cdot \mathrm{g}^{-1}$, and $\gamma_{M}=1 \mathrm{~m}^{3} \cdot \mathrm{g}^{-1}$. Choosing accordingly the incident light intensity $I_{\text {in }}$ and the setpoint $I_{\text {out }}^{*}$, control law (13) allows to select each species.

Finally, we test our approach in a more realistic simulation, that is ten species with different attenuation coefficients (see Figure 5). The closed-loop culture allows to select the species that maximizes $\int_{I_{\text {out }}^{*}}^{I_{\text {in }}} f_{i}(I) d I$. Since the best competitor and some other species have growth functions close to each other in the range $\left[I_{\text {out }}^{*} ; I_{\text {in }}\right]$, the selection process requires a long time. Nevertheless, it appears that the worst competitors are rapidly excluded, so this approach can be used to select rapidly a subset of species of interest. Note that such aspect of "long run coexistence" has been tackled in Rapaport et al. (2009) for competition under substrate limitation using slow-fast characterization. 

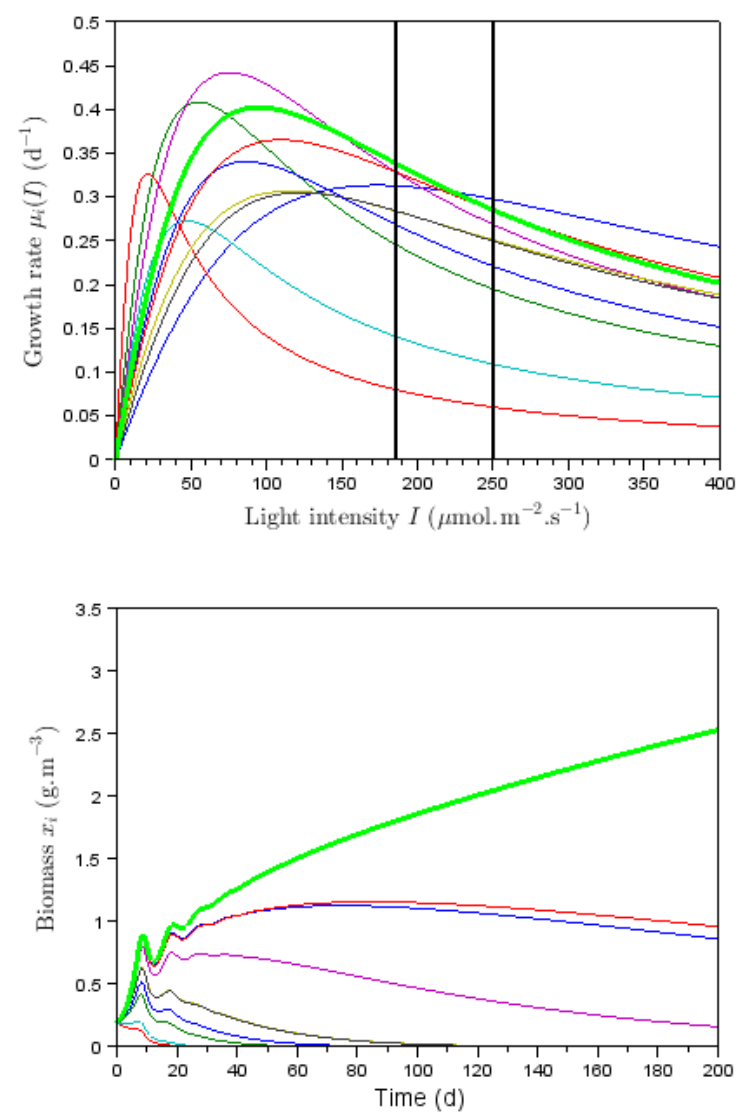

Fig. 5. Simulations of competition in closed-loop in a more realistic framework (10 species with different attenuation coefficients. Top: specific growth rates of the ten species (vertical lines: $I_{\text {in }}$ and $I_{\text {out }}^{*}$ ). Bottom: time evolution of species concentrations.

Finally, from intensive numerical simulations, it seems that Hypothesis 4 (i) is not necessary for the selection process. Proof of Theorem 5 without this hypothesis is under investigation.

\section{CONCLUSION}

In this paper, we have proposed a nonlinear adaptive control which regulates the light intensity at the bottom of the water column in monoculture. The resulting controller was further applied for the case of a culture with $n$ species. The control law allows the selection of the strain with the maximum growth rate for a given range of light intensity. This is of particular interest for optimizing biomass production as species adapted to daylight levels and reactor configurations can be used. In the future, the proof of concept of such principles will be carried out experimentally.

\section{REFERENCES}

Bastin, G. and Dochain, D. (1990). On-line estimation and adaptive control of bioreactors. Elsevier, New York.

Bernard, O. (2011). Hurdles and challenges for modelling and control of microalgae for co2 mitigation and biofuel production. Journal of Process Control, 21, 1378-1389.
Bernard, O., Masci, P., and Sciandra, A. (2009). A photobioreactor model in nitrogen limited conditions. In Proceedings of the $6^{\text {th }}$ Conference on Mathematical Modelling. Vienna.

Chisti, Y. (2007). Biodiesel from microalgae. Biotechnology Advances, 25, 294-306.

Gerla, D., Mooij, W., and Huisman, J. (2011). Photoinhibition and the assembly of light-limited phytoplankton communities. Oikos, 120(3), 359-368.

Hansen, S.R. and Hubbell, S.P. (1980). Single-nutrient microbial competition. Science, 207(28), 1491-1493.

Huisman, J., Jonker, R., Zonneveld, C., and Weissing, F. (1999). Competition for light between phytoplankton species: experimental tests of mechanistic theory. Ecology, 80(1), 211-222.

Huisman, J. and Weissing, F. (1994). Light-limited growth and competition for light in well-mixed aquatic environments: an elementary model. Ecology, 75, 507-520.

Khalil, H. (2002). Nonlinear systems. Prentice Hall, third edition.

Mailleret, L., Bernard, O., and Steyer, J.P. (2004). Robust nonlinear adaptive control for bioreactors with unknown kinetics. Automatica, 40:8, 365-383.

Mairet, F., Moisan, M., and Bernard, O. (2010). Interval observer-based estimator of specific growth rate in bioreactors. Journal Européen des Systèmes Automatisés, 44(4-5), 493-507.

Masci, P., Bernard, O., and Grognard, F. (2008). Continuous selection of the fastest growing species in the chemostat. In 17th IFAC World Congress, Seoul, Korea.

Masci, P., Bernard, O., Grognard, F., Latrille, E., Sorba, J., and Steyer, J. (2009). Driving competition in a complex ecosystem: application to anaerobic digestion. In 10th ECC conference, Budapest, Hungary.

Perrier, M., de Azevedo, S.F., Ferreira, E., and Dochain, D. (2000). Tuning of observer-based estimators: theory and application to the on-line estimation of kinetic parameters. Control Engineering Practice, 8(4), 377388.

Rapaport, A., Dochain, D., and Harmand, J. (2009). Long run coexistence in the chemostat with multiple species. Journal of theoretical biology, 257(2), 252-259.

Sbarciog, M. and Vande Wouwer, A. (2012). Some considerations about control of multi-species anaerobic digestion systems. In Proceedings of the 7th Vienna International Conference on Mathematical Modelling (MATH$M O D)$. Vienna, Austria.

Smith, H.L. and Waltman, P. (1995). The theory of the chemostat: dynamics of microbial competition. Cambridge University Press.

Spolaore, P., Joannis-Cassan, C., Duran, E., and Isambert, A. (2006). Commercial applications of microalgae. Journal of bioscience and bioengineering, 101(2), 87-96.

Stomp, M., Huisman, J., De Jongh, F., Veraart, A., Gerla, D., Rijkeboer, M., Ibelings, B., Wollenzien, U., and Stal, L. (2004). Adaptive divergence in pigment composition promotes phytoplankton biodiversity. Nature, 432(7013), 104-107.

Weissing, F. and Huisman, J. (1994). Growth and competition in a light gradient. Journal of theoretical biology, $168,323-336$.

Wijffels, R. and Barbosa, M. (2010). An Outlook on Microalgal Biofuels. Science, 329(5993), 796-799. 